\title{
HUBUNGAN ANTARA BEBERAPA JENIS HIMPUNAN KABUR DAN ENTROPI PADA HIMPUNAN LEMBUT KABUR INTUISIONISTIK
}

\author{
Raisatul Mardhiyah, Nova Noliza Bakar \\ Program Studi S1 Matematika, \\ Fakultas Matematika dan Ilmu Pengetahuan Alam, Universitas Andalas, \\ Kampus UNAND Limau Manis Padang, Indonesia. \\ email : raisatulmardhiyah@gmail.com
}

\begin{abstract}
Abstrak. Himpunan lembut yang diperkenalkan oleh Molodsov(1986) adalah suatu alat matematika baru untuk mengatasi ketidakpastian di bidang matematika yang tidak dapat diselesaikan secara biasa [10]. Himpunan Kabur diperkenalkan oleh L.A Zadeh (1965) [11]. Penggabungan antara teori himpunan lembut dan himpunan kabur dinamakan teori himpunan lembut kabur [8]. Himpunan kabur diperluas menjadi beberapa bentuk himpunan-himpunan kabur seperti himpunan kabur intuisionistik [1] dan himpunan kabur bernilai interval [8]. Hubungan antara beberapa jenis himpunan kabur adalah salah satu topik yang menarik untuk dikaji pada teori himpunan kabur ini. Pada tulisan ini akan dibahas bagaimana hubungan antara beberapa jenis himpunan kabur serta sifat-sifatnya, dan bagaimana sifat dan contoh entropi pada himpunan lembut kabur intuisionistik.
\end{abstract}

Kata Kunci: Himpunan kabur, Himpunan lembut kabur intuisionistik, Entropi.

\section{Pendahuluan}

Hubungan antara beberapa jenis himpunan kabur adalah salah satu topik yang menarik untuk dikaji pada teori himpunan kabur. Beberapa hubungan antara koleksi-koleksi himpunan-himpunan kabur yaitu: a) hubungan antara koleksi himpunan kabur bernilai interval dan koleksi himpunan kabur intuisionistik [3], b) hubungan antara koleksi himpunan kabur bernilai interval dan koleksi himpunan kabur [4], c) hubungan antara koleksi himpunan kabur intuisionistik dan koleksi himpunan kabur [4], dan d) hubungan antara koleksi himpunan lembut kabur intuisionistik dan koleksi himpunan lembut kabur [7], serta entropi pada himpunan lembut kabur intuisionistik [7]. Pada artikel ini akan dibahas kembali hal-hal di atas secara lebih terperinci. 


\section{Konsep-Konsep Dasar Teori Himpunan Lembut Kabur Intuisionistik}

\subsection{Himpunan Kabur}

Himpunan kabur pertama kali diperkenalkan oleh L.A Zadeh [11] pada tahun 1965. Himpunan kabur memiliki derajat keanggotaan yang terletak dalam interval $[0,1]$ dan fungsi keanggotaannya $\mu_{A}: U \rightarrow[0,1]$, dimana $U$ merupakan himpunan semesta.

Definisi 2.1. [11] Misalkan U adalah himpunan semesta yang tak kosong. Suatu himpunan kabur A atas $U$ didefinisikan sebagai berikut:

$$
A=\left\{\left(x, \mu_{A}(x)\right) \mid x \in U\right\}
$$

dimana $\mu_{A}: U \rightarrow[0,1]$, dan $\mu_{A}(x)$ disebut derajat keanggotaan dari himpunan kabur A.

\subsection{Himpunan Lembut}

Definisi 2.2. [10] Misalkan $U$ adalah himpunan semesta yang tak kosong, E adalah suatu himpunan parameter, dan $P(U)$ adalah koleksi dari seluruh himpunan bagian atas U. Pasangan $\langle L, E\rangle$ disebut suatu himpunan lembut atas $U$ jika dan hanya jika $L$ adalah suatu pemetaan yang diberikan oleh $L: E \rightarrow P(U)$, yang dapat dinyatakan sebagai himpunan pasangan terurut yang berbentuk:

$$
\langle L, E\rangle=\{(\varepsilon, L(\varepsilon)) \mid \varepsilon \in E, L(\varepsilon) \in P(U)\} .
$$

\subsection{Himpunan Lembut Kabur}

Himpunan lembut kabur merupakan penggabungan teori himpunan lembut dan teori himpunan kabur.

Definisi 2.3 [8] Misalkan U adalah himpunan semesta yang tak kosong, E adalah suatu himpunan parameter, dan $A \subseteq E$. Pasangan $\langle F, A\rangle$ disebut himpunan lembut kabur jika $F$ adalah pemetaan yang diberikan oleh $F: A \rightarrow \mathcal{F S}(\mathcal{U})$, yang dapat dinyatakan sebagai himpunan pasangan terurut yang berbentuk:

$$
\langle F, A\rangle=\{(\alpha, F(\alpha)) \mid \alpha \in A, F(\alpha) \in \mathcal{F} \mathcal{S}(\mathcal{U})\} .
$$

dengan $\mathcal{F S}(\mathcal{U})$ adalah himpunan dari seluruh himpunan kabur atas $U$.

\subsection{Himpunan Kabur Intuisionistik}

Himpunan kabur intuisiontistik merupakan himpunan kabur yang memperhitungkan nilai keanggotaan dan nilai ketidakanggotaan.

Definisi 2.4 [1] Misalkan U adalah himpunan semesta yang tak kosong. Suatu himpunan kabur intuisionistik $\hat{A}$ atas $U$ didefinisikan sebagai berikut:

$$
\hat{A}=\left\{\left(x, \mu_{\hat{A}}(x), \gamma_{\hat{A}}(x)\right) \mid x \in U\right\}
$$


dimana $\mu_{\hat{A}}: U \rightarrow[0,1]$, dan $\gamma_{\hat{A}}: U \rightarrow[0,1]$ berturut-turut menyatakan fungsi keanggotaan dan ketidakanggotaan pada himpunan kabur intuisionistik $\hat{A}$, dan untuk setiap $x \in U$ harus memenuhi $0 \leq \mu_{\hat{A}}(x)+\gamma_{\hat{A}}(x) \leq 1$ dan $\pi_{\hat{A}}(x)=1-\mu_{\hat{A}}(x)-\gamma_{\hat{A}}(x)$ disebut derajat keragu-raguan dari $x \in U$ pada himpunan kabur intuisionistik $\hat{A}$.

\subsection{Himpunan Lembut Kabur Intuisionistik}

Definisi 2.5 [8] Misalkan U adalah himpunan semesta yang tak kosong, E adalah himpunan parameter, dan $\mathcal{I} \mathcal{F} \mathcal{S}(\mathcal{U})$ adalah himpunan dari seluruh himpunan kabur intuisionistik atas U. Pasangan $\langle\bar{F}, \bar{E}\rangle$ disebut himpunan lembut kabur intuisionistik atas $U$, jika $\bar{F}$ adalah pemetaan yang diberikan oleh $\bar{F}: \bar{E} \rightarrow \mathcal{I} \mathcal{F S}(\mathcal{U})$. Suatu himpunan lembut kabur intuisionistik atas $U$ dapat didefinisikan sebagai berikut:

$$
\langle\bar{F}, \bar{E}\rangle=\{(e, \bar{F}(e)) \mid e \in E, \bar{F}(e) \in \mathcal{I} \mathcal{F} \mathcal{S}(\mathcal{U})\}
$$

\subsection{Himpunan Kabur Bernilai Interval}

Definisi 2.6 [8] Suatu himpunan kabur bernilai interval $\bar{A}$ atas himpunan semesta $U$ adalah suatu himpunan yang terkait dengan pemetaan yang diberikan oleh $M_{\bar{A}}$ : $U \rightarrow \operatorname{int}[0,1]$, dimana int [0,1] adalah himpunan dari seluruh subinterval tertutup dari interval [0,1], yang dapat ditulis dalam bentuk:

$$
\bar{A}=\left\{\left(x, M_{\bar{A}}(x)\right) \mid x \in U\right\}=\left\{\left(x,\left[M_{\bar{A}}^{L}(x), M_{\bar{A}}^{U}(x)\right]\right) \mid x \in U\right\},
$$

dengan $M_{\bar{A}}(x)=\left[M_{\bar{A}}^{L}(x), M_{\bar{A}}^{U}(x)\right]$,

dimana $M_{\bar{A}}(x)$ disebut derajat keanggotaan dari suatu elemen $x \in U$,

dan $M_{\bar{A}}^{L}(x), M_{\bar{A}}^{U}(x)$ berturut-turut menyatakan derajat keanggotaan bawah dan atas dari $x \in U$, yang memenuhi kondisi $0 \leq M_{A}{ }^{L}(x) \leq M_{A}{ }^{U}(x) \leq 1$.

\section{Hubungan antara Koleksi Himpunan Lembut Kabur Intuisionistik dan Koleksi Himpunan Lembut Kabur}

Pada bagian ini akan diperkenalkan suatu bentuk hubungan antara koleksi himpunan-himpunan lembut kabur intuisionistik dan koleksi himpunan-himpunan lembut kabur.

Definisi 3.1. [7] Misalkan $E=\left\{e_{1}, e_{2}, e_{3}, \ldots, e_{n}\right\}$ adalah himpunan parameter. Bukan himpunan dari $E$ dinotasikan dengan $\rceil E$ didefinisikan sebagai $\rceil E=$ $\left\{\neg e_{1}, \neg e_{2}, \neg e_{3}, \ldots, \neg e_{n}\right\}$, dimana $\neg e_{i}=$ bukan $e_{i}$ dan $\neg \neg e_{i}=e_{i}, \forall e_{i} \in E$.

Definisi 3.2. [7] Komplemen dari himpunan lembut kabur intuisionistik $\omega=$ $\langle F, E\rangle$, dinotasikan dengan $\langle F, E\rangle^{c}$, dan didefinisikan dengan $\left.(\langle F, E\rangle)^{c}=\left\langle F^{c},\right\rceil E\right\rangle$, dimana:

$\left.F^{c}:\right\rceil E \rightarrow \mathcal{I F} \mathcal{S}(\mathcal{U})$ adalah pemetaan yang diberikan oleh:

$$
\begin{aligned}
F^{c}(\neg \varepsilon) & =\left\{\left(x,\left(\gamma_{F^{c}(\neg \varepsilon)}(x), \mu_{F^{c}(\neg \varepsilon)}(x)\right)\right) \mid x \in U\right\} \\
& =\left\{\left(x,\left(\gamma_{F(\neg \neg \varepsilon)}(x), \mu_{F(\neg \neg \varepsilon)}(x)\right)\right) \mid x \in U\right\} \\
& \left.=\left\{\left(x,\left(\gamma_{F(\varepsilon)}(x), \mu_{F(\varepsilon}(x)\right)\right) \mid x \in U\right\}, \forall(\neg \varepsilon) \in(\rceil E\right) .
\end{aligned}
$$


Definisi 3.3. [7] Misalkan $\omega=\langle F, E\rangle$ adalah himpunan lembut kabur intuisionistik atas $U$, dimana $\omega \in \mathcal{I} \mathcal{F} \mathcal{S} \mathcal{S}(\mathcal{U})$. Himpunan $\omega$ disebut intuisionistik lengkap, jika $\mu_{F(\varepsilon)}(x)=\gamma_{F \varepsilon)}(x)=0, \forall \varepsilon \in E$ dan $\forall x \in U$.

Definisi 3.4. [7] Untuk setiap $\alpha \in[0,1]$ didefinisikan suatu pemetaan $f_{\alpha}$ dari koleksi himpunan-himmpunan lembut kabur intuisionistik ke koleksi himpunanhimpunan lembut kabur yang diberikan sebagai berikut:

$f_{\alpha}: \mathcal{I F S S}(\mathcal{U}) \rightarrow \mathcal{F S S}(\mathcal{U})$

$\langle F, E\rangle \rightarrow f_{\alpha}(\langle F, E\rangle)=\left\langle F_{\alpha}, E\right\rangle$ dimana $F_{\alpha}$ didefinisikan sebagai berikut:

misal $F(\varepsilon)=\left\{\left(x, \mu_{F(\varepsilon)}(x), \gamma_{F(\varepsilon)}(x)\right) \mid x \in U\right\}, \forall \varepsilon \in E$.

$$
\begin{aligned}
F_{\alpha}(\varepsilon)=f_{\alpha}(F(\varepsilon))(x) & \left.=f_{\alpha}\left(\left\{\left(x, \mu_{F(\varepsilon)}(x), \gamma_{F(\varepsilon)}(x)\right)\right) \mid x \in U\right\}\right) \\
& =\left\{\left(x, \mu_{F(\varepsilon)}(x)+\alpha \pi_{F(\varepsilon)}(x), 1-\mu_{F(\varepsilon)}(x)-\alpha \pi_{F(\varepsilon)}(x)\right\rangle \mid x \in U\right\} .
\end{aligned}
$$

Teorema 3.5. [7] Untuk sebarang $\alpha, \beta \in[0,1]$ dan $\omega, \sigma \in \mathcal{I} \mathcal{F S S}(\mathcal{U})$ berlaku sifatsifat sebagai berikut:

(a) jika $\alpha \leq \beta$, maka $f_{\alpha}(\omega) \Subset f_{\beta}(\omega)$,

(b) jika $\omega \Subset \sigma$, maka $f_{\alpha}(\omega) \Subset f_{\alpha}(\sigma)$,

(c) $f_{\alpha}\left(f_{\beta}(\omega)\right)=f_{\beta}(\omega)$,

(d) $\left(f_{\alpha}\left(\omega^{c}\right)\right)^{c}=f_{1-\alpha}(\omega)$.

\section{Bukti :}

Misal $\omega=\langle F, E\rangle$ dan $\sigma=\langle G, E\rangle$ adalah himpunan lembut kabur intuisionistik.

Misal $f_{\alpha}(\omega)=f_{\alpha}(\{\langle F, E\rangle\})=\left\langle F_{\alpha}, E\right\rangle$, dimana

$$
\begin{aligned}
F_{\alpha}(\varepsilon)=f_{\alpha}(F(\varepsilon)) & =f_{\alpha}\left(\left\{\left(x, \mu_{F(\varepsilon)}(x), \gamma_{F(\varepsilon)}(x)\right) \mid x \in U\right\}\right) \\
& =\left\{\left(x, \mu_{F(\varepsilon)}(x)+\alpha \pi_{F(\varepsilon)}(x), 1-\mu_{F(\varepsilon)}(x)-\alpha \pi_{F(\varepsilon)}(x)\right) \mid x \in U\right\}, \forall \varepsilon \in E .
\end{aligned}
$$

Misal $f_{\beta}(\omega)=f_{\beta}(\{\langle F, E\rangle\})=\left\langle F_{\beta}, E\right\rangle$, dimana

$$
\begin{aligned}
F_{\beta}(\varepsilon)=f_{\beta}(F(\varepsilon)) & =f_{\beta}\left(\left\{\left(x, \mu_{F(\varepsilon)}(x), \gamma_{F(\varepsilon)}(x)\right) \mid x \in U\right\}\right) \\
& =\left\{\left(x, \mu_{F(\varepsilon)}(x)+\beta \pi_{F(\varepsilon)}(x), 1-\mu_{F(\varepsilon)}(x)-\beta \pi_{F(\varepsilon)}(x)\right) \mid x \in U\right\}, \forall \varepsilon \in E .
\end{aligned}
$$

(a) Misal $\alpha \leq \beta$, akan dibuktikan $f_{\alpha}(\omega) \Subset f_{\beta}(\omega)$.

Dari Definisi 2.3.1(a), jelas $E \subseteq E$.

Selanjutnya, akan ditunjukkan $\forall \varepsilon \in E, F_{\alpha}(\varepsilon) \subseteq F_{\beta}(\varepsilon)$.

Untuk ini cukup dengan menunjukkan $\forall x \in U, \varepsilon \in E, \mu_{F_{\alpha}(\varepsilon)}(x) \leq \mu_{F_{\beta}(\varepsilon)}(x)$.

Karena $\mu_{F_{\alpha}(\varepsilon)}(x) \leq \mu_{F \beta(\varepsilon)}(x)$, maka terbukti bahwa $f_{\alpha}(\omega) \Subset f_{\beta}(\omega)$.

(b) Misal $\omega \Subset \sigma$, akan dibuktikan $f_{\alpha}(\omega) \Subset f_{\alpha}(\sigma)$.

Misal $f_{\alpha}(\sigma)=f_{\alpha}\{\langle G, E\rangle\}=\left\langle G_{\alpha}, E\right\rangle$, dimana

$$
\begin{aligned}
G_{\alpha}(\varepsilon)=f_{\alpha}(G(\varepsilon)) & =f_{\alpha}\left(\left\{\left(x, \mu_{G(\varepsilon)}, \gamma_{G(\varepsilon)}\right) \mid x \in U\right\}\right) \\
& =\left\{\left(x, \mu_{G(\varepsilon)}+\alpha \pi_{G(\varepsilon)}, 1-\mu_{G(\varepsilon)}-\alpha \pi_{G(\varepsilon)}\right) \mid x \in U\right\}, \forall \varepsilon \in E .
\end{aligned}
$$

Dari Definisi 2.3.1(a), jelas $E \subseteq E$.

Selanjutnya, akan ditunjukkan $\forall \varepsilon \in E$, akan ditunjukkan $F_{\alpha}(\varepsilon) \subseteq G_{\alpha}(\varepsilon)$.

Untuk ini cukup dengan menunjukkan $\forall x \in U, \varepsilon \in E, \omega \Subset \sigma, \mu_{F_{\alpha}(\varepsilon)}(x) \leq$ $\mu_{G_{\alpha}(\varepsilon)}(x)$.

Karena $\mu_{F_{\alpha}(\varepsilon)}(x) \leq \mu_{G \alpha(\varepsilon)}(x)$, maka terbukti bahwa $f_{\alpha}(\omega) \Subset f_{\alpha}(\sigma)$. 
(c) Akan dibuktikan $f_{\alpha}\left(f_{\beta}(\omega)\right)=f_{\beta}(\omega)$.

Misal $f_{\alpha}\left(f_{\beta}(\omega)\right)=f_{\alpha}\left(\left\langle F_{\beta}, E\right\rangle\right)=\left\langle\left(F_{\beta}\right)_{\alpha}, E\right\rangle$, dimana

$\left(F_{\beta}\right)_{\alpha}(\varepsilon)=f_{\alpha}\left(F_{\beta}(\varepsilon)\right)=f_{\alpha}\left(f_{\beta}(F(\varepsilon))\right), \forall \varepsilon \in E$.

Jelas $\mathrm{E}=\mathrm{E}$.

Karena $f_{\beta}(F(\varepsilon))=\left\{\left(x, \mu_{F(\varepsilon)}(x)+\beta \pi_{F(\varepsilon)}(x), 1-\mu_{F(\varepsilon)}(x)+\beta \pi_{F(\varepsilon)}(x)\right) \mid x \epsilon U\right\}$, maka $\forall \varepsilon \in E$,

$$
\begin{aligned}
f_{\alpha}\left(f_{\beta}(F(\varepsilon))=\right. & f_{\alpha}\left(\left\{\left(x, \mu_{F(\varepsilon)}(x)+\beta \pi_{F(\varepsilon)}(x), 1-\mu_{F(\varepsilon)}(x)+\beta \pi_{F(\varepsilon)}(x)\right) \mid x \in U\right\}\right) . \\
= & \left(\left\{\left(x,\left(\mu_{F(\varepsilon)}(x)+\beta \pi_{F(\varepsilon)}(x)\right)+\alpha\left(1-\mu_{F(\varepsilon)}(x)+\beta \pi_{F(\varepsilon)}(x)\right)-(1-\right.\right.\right. \\
& \left.\mu_{F(\varepsilon)}(x)-\beta \pi_{F(\varepsilon)}(x)\right), 1-\left(\mu_{F(\varepsilon)}(x)+\beta \pi_{F(\varepsilon)}(x)\right)+\alpha\left(1-\mu_{F(\varepsilon)}(x)\right. \\
& \left.\left.\left.\left.+\beta \pi_{F(\varepsilon)}(x)\right)-\left(1-\mu_{F(\varepsilon)}(x)-\beta \pi_{F(\varepsilon)}(x)\right)\right) \mid x \in U\right\}\right) . \\
= & \left(\left\{\left(x, \mu_{F(\varepsilon)}(x)+\beta \pi_{F(\varepsilon)}(x), 1-\mu_{F(\varepsilon)}(x)-\beta \pi_{F(\varepsilon)}(x)\right) \mid \in U\right\}\right) .
\end{aligned}
$$

Karena $f_{\alpha}\left(f_{\beta}(F(\varepsilon))\right)=f_{\beta}(F(\varepsilon))$, maka terbukti bahwa $f_{\alpha}\left(f_{\beta}(\omega)\right)=f_{\beta}(\omega)$.

(d) Akan dibuktikan $\left(f_{\alpha}\left(\omega^{c}\right)\right)^{c}=f_{1-\alpha}(\omega)$.

Misal $\left.\omega^{c}=\langle F, E\rangle^{c}=\left\langle F^{c},\right\rceil E\right\rangle$, dimana

$\left.F^{c}(\varepsilon)=\left\{\left(x, \gamma_{F(\neg \varepsilon)}(x), \mu_{F(\neg \varepsilon)}(x)\right) \mid x \in U\right\}, \forall \varepsilon \in\right\rceil E$.

Karena $\left.\left.f_{\alpha}\left(\omega^{c}\right)=f_{\alpha}\left(\left\langle F^{c},\right\rceil E\right\rangle\right)=\left\langle\left(F^{c}\right)_{\alpha},\right\rceil E\right\rangle$,

$$
\begin{aligned}
\left(F^{c}\right)_{\alpha}(\varepsilon)=f_{\alpha}\left(F^{c}(\varepsilon)\right)= & f_{\alpha}\left(\left\{\left(x, \gamma_{F(\neg \varepsilon)}(x), \mu_{F(\neg \varepsilon)}(x)\right) \mid x \in U\right\}\right) . \\
= & \left\{\left(x, \gamma_{F(\neg \varepsilon)}(x)+\alpha\left(1-\gamma_{F(\neg \varepsilon)}(x)-\mu_{F(\neg \varepsilon)}(x)\right),\right.\right. \\
& \left.1-\left(\gamma_{F(\neg \varepsilon)}(x)+\alpha\left(1-\gamma_{F(\neg \varepsilon)}(x)-\mu_{F(\neg \varepsilon)}(x)\right)\right) \mid x \in U\right\}, \\
& \forall \varepsilon \in\rceil E .
\end{aligned}
$$

Selanjutnya, karena $\left(f_{\alpha}\left(\omega^{c}\right)\right)^{c} \in \mathcal{I} \mathcal{F} \mathcal{S} \mathcal{S}(\mathcal{U})$, maka $\left.\left.\left.\left(f_{\alpha}\left(\omega^{c}\right)\right)^{c}=\left\langle\left(F^{c}\right)_{\alpha},\right\rceil E\right\rangle^{c}=\left\langle\left(\left(F^{c}\right)_{\alpha}\right)^{c},\right\rceil\right\rceil E\right\rangle=\left\langle\left(\left(F^{c}\right)_{\alpha}\right)^{c}, E\right\rangle$.

Akibatnya $\forall \varepsilon \in E$, diperoleh

$$
\begin{aligned}
\left(\left(F^{c}\right)_{\alpha}\right)^{c}(\varepsilon)= & \left\{\left(x, 1-\left(\gamma_{F(\neg \neg \varepsilon)}(x)+\alpha\left(1-\gamma_{F(\neg \neg \varepsilon)}(x)-\mu_{F(\neg \neg \varepsilon)}(x)\right)\right),\right.\right. \\
& \left.\left.\gamma_{F(\neg \neg \varepsilon)}(x)+\alpha\left(1-\gamma_{F(\neg \neg \varepsilon)}(x)-\mu_{F(\neg \neg \varepsilon)}(x)\right)\right) \mid x \in U\right\} . \\
= & \left\{\left(x, 1-\gamma_{F(\varepsilon)}-\alpha+\alpha \gamma_{F(\varepsilon)}(x)+\alpha \mu_{F(\varepsilon)}(x),\right.\right. \\
& \left.\gamma_{F(\varepsilon)}(x)+\alpha-\alpha \gamma_{F(\varepsilon)}(x)-\alpha \mu_{F(\varepsilon)}(x) \mid x \in U\right\} .
\end{aligned}
$$

Misal $f_{1-\alpha}(\omega)=f_{1-\alpha}(\langle F, E\rangle)=\left\langle F_{1-\alpha}, E\right\rangle$, dimana

$F_{1-\alpha}(\varepsilon)=f_{1-\alpha}(F(\varepsilon))$.

Jelas $E=E$, maka

$$
\begin{aligned}
f_{1-\alpha}(F(\varepsilon))= & f_{1-\alpha}\left(\left\{\left(x, \mu_{F(\varepsilon)}(x), \gamma_{F(\varepsilon)}(x)\right) \mid x \in U\right\}\right) . \\
= & \left\{\left(x, \mu_{F(\varepsilon)}(x)+\alpha \pi_{F(\varepsilon)}(x), 1-\mu_{F(\varepsilon)}(x)-\alpha \pi_{F(\varepsilon)}(x)\right) \mid x \in U\right\} . \\
= & \left\{\left(x, \mu_{F(\varepsilon)}(x)+(1-\alpha)\left(1-\mu_{F(\varepsilon)}(x)-\gamma_{F(\varepsilon)}(x),\right.\right.\right. \\
& \left.1-\mu_{F(\varepsilon)}(x)-(1-\alpha)\left(1-\mu_{F(\varepsilon)}(x)-\gamma_{F(\varepsilon)}(x)\right) \mid x \in U\right\} . \\
= & \left\{\left(x, 1-\gamma_{F(\varepsilon)}(x)-\alpha+\alpha \mu_{F(\varepsilon)}(x)+\alpha \gamma_{F(\varepsilon)}(x), \gamma_{F(\varepsilon)}(x)\right.\right. \\
& \left.\left.+\alpha-\alpha \mu_{F(\varepsilon)}(x)-\alpha \gamma_{F(\varepsilon)}(x)\right) \mid x \in U\right\} .
\end{aligned}
$$

Karena $\left(\left(F^{c}\right)_{\alpha}\right)^{c}(\varepsilon)=F_{1-\alpha}(\varepsilon)$, maka terbukti $\left(f_{\alpha}\left(\omega^{c}\right)\right)^{c}=f_{1-\alpha}(\omega)$.

\section{Entropi pada Himpunan Lembut Kabur Intuisionistik}

Pada bagian ini akan diperkenalkan suatu entropi pada himpunan lembut kabur instuisionisti dan sifat-sifat terkaitnya. 
Definisi 4.1. [7] Entropi pada himpunan lembut kabur intuisionistik, didefini-sikan sebagai suatu pemetaan $I: \mathcal{I} \mathcal{F S S}(\mathcal{U}) \rightarrow \boldsymbol{R}^{+}=[0, \infty)$, berlaku sifat-sifat sebagai berikut:

(a) $I(\omega)=0$ jika dan hanya jika $\omega \in \mathcal{F} \mathcal{S S}(\mathcal{U})$,

(b) misal $\omega=\langle F, E\rangle=\left[a_{i j}\right]_{m \times n}, I(\omega)=$ mn jika dan hanya jika $\mu_{F\left(\varepsilon_{j}\right)}\left(u_{i}\right)=$ $\gamma_{F\left(\varepsilon_{j}\right)}\left(u_{i}\right)=0, \forall \varepsilon_{j} \in E, \forall u_{i} \in U$, dimana $a_{i j}=\left(\mu_{F\left(\varepsilon_{j}\right)}\left(u_{i}\right), \gamma_{F\left(\varepsilon_{j}\right)}\left(u_{i}\right)\right)$,

(c) $I(\omega)=I\left(\omega^{c}\right)$ untuk semua $\omega \in \mathcal{I} \mathcal{F S S}(\mathcal{U})$,

(d) jika $\omega \preceq \sigma$, maka $I(\omega) \geq I(\sigma)$, atau $I(\omega)$ adalah maksimum, dimana $\omega=\langle F, E\rangle$ dan $\sigma=\langle G, E\rangle$.

Berikut ini akan diberikan suatu fungsi yang akan dikaitkan dengan konsep entropi pada himpunan lembut kabur intuisionistik [5].

Diberikan himpunan:

$$
D=\{(x, y) \mid x, y \in[0,1], x+y \leq 1\}
$$

dan $\Phi: D \rightarrow[0,1]$ yang memenuhi kondisi:

(a) $\Phi_{D}(x, y)=1$ jika dan hanya jika $x+y=1$,

(b) $\Phi_{D}(x, y)=0$ jika dan hanya jika $x=0=y$,

(c) $\Phi_{D}(x, y)=\Phi_{D}(y, x)$,

(d) jika $x \leq x^{\prime}$ dan $y \leq y^{\prime}$ maka $\Phi_{D}(x, y) \leq \Phi_{D}\left(x^{\prime}, y^{\prime}\right)$.

Teorema 4.2. [7] Misal I: $\mathcal{I} \mathcal{F S S}(\mathcal{U}) \rightarrow \boldsymbol{R}^{+}$dan $\omega=\langle F, E\rangle=\left[a_{i j}\right]_{m \times n} \in$ $\mathcal{I F S S}(\mathcal{U})$

Jika $I(\omega)=\sum_{j=1}^{n} \sum_{i=1}^{m}\left(1-\Phi\left(\mu_{F\left(\varepsilon_{j}\right)}\left(u_{i}\right), \gamma_{F\left(\varepsilon_{j}\right)}\left(u_{i}\right)\right)\right)$,

dimana $\Phi$ memenuhi kondisi (a)-(d) di atas, maka I merupakan suatu entropi.

\section{Bukti:}

Ambil $\omega \in \mathcal{I} \mathcal{F} \mathcal{S} \mathcal{S}(\mathcal{U})$.

Akan dibuktikan bahwa $I(\omega)=\sum_{j=1}^{n} \sum_{i=1}^{m}\left(1-\Phi\left(\mu_{F\left(\varepsilon_{j}\right)}\left(u_{i}\right), \gamma_{F\left(\varepsilon_{j}\right)}\left(u_{i}\right)\right)\right)$ adalah sebuah entropi, dengan $\Phi$ memenuhi kondisi (a), (b), (c), dan (d).

(a) Akan ditunjukkan $I(\omega)=0$ jika dan hanya jika $\omega \in \mathcal{F} \mathcal{S} \mathcal{S}(\mathcal{U})$.

$(\Longrightarrow)$ Misal $I(\omega)=0$, maka akan dibuktikan $\omega \in \mathcal{F} \mathcal{S S}(\mathcal{U})$. Berdasarkan kondisi (a), diperoleh $\mu_{F\left(\varepsilon_{j}\right)}\left(u_{i}\right)+\gamma_{F\left(\varepsilon_{j}\right)}\left(u_{i}\right)=1$, sehingga $\gamma_{F\left(\varepsilon_{j}\right)}\left(u_{i}\right)=1-\mu_{F\left(\varepsilon_{j}\right)}\left(u_{i}\right)$.

Akibatnya $\omega=\left\{\left(u_{i}, \mu_{F\left(\varepsilon_{j}\right)}\left(u_{i}\right), 1-\mu_{F\left(\varepsilon_{j}\right)}\left(u_{i}\right)\right) \mid \varepsilon_{j} \in E, u_{i} \in U\right\}$ atau $\omega \in \mathcal{F} \mathcal{S S}(\mathcal{U})$.

$(\Longleftarrow)$ Misal $\omega \in \mathcal{F} \mathcal{S S}(\mathcal{U})$, maka akan dibuktikan $I(\omega)=0$.

Karena $\omega=\left\{\left(u i, \mu_{F\left(\varepsilon_{j}\right)}\left(u_{i}\right), 1-\mu_{F\left(\varepsilon_{j}\right)}\left(u_{i}\right)\right) \mid \varepsilon_{j} \in E, u_{i} \in U\right\}$, dimana $1-\mu_{F\left(\varepsilon_{j}\right)}\left(u_{i}\right)=\gamma_{F\left(\varepsilon_{j}\right)}\left(u_{i}\right)$, maka $\mu_{F\left(\varepsilon_{j}\right)}\left(u_{i}\right)+\gamma_{F\left(\varepsilon_{j}\right)}\left(u_{i}\right)=1$.

Berdasarkan kondisi (a), diperoleh $\Phi\left(\mu_{F\left(\varepsilon_{j}\right)}\left(u_{i}\right), \gamma_{F\left(\varepsilon_{j}\right)}\left(u_{i}\right)\right)=1$, sehingga dieroleh $I(\omega)=0$.

(b) Akan ditunjukkan $I(\omega)=m n$, jika dan hanya jika $\mu_{F\left(\varepsilon_{j}\right)}\left(u_{i}\right)=\gamma_{F\left(\varepsilon_{j}\right)}\left(u_{i}\right)=$ $0, \forall \varepsilon_{j} \in E, \forall u_{i} \in U$. 
$(\Longrightarrow)$ Ambil $\omega \in \mathcal{I} \mathcal{F} \mathcal{S S}(\mathcal{U})$ dan $I(\omega)=m n$,

maka akan dibuktikan $\mu_{F\left(\varepsilon_{j}\right)}\left(u_{i}\right)=\gamma_{F\left(\varepsilon_{j}\right)}\left(u_{i}\right)=0$.

$\left.\left.I\left[\left(\mu_{F\left(\varepsilon_{j}\right.}\right)\left(u_{i}\right)\right), \gamma_{F\left(\varepsilon_{j}\right.}\right)\left(u_{i}\right)\right]=m n$

Berdasarkan kondisi (b), diperoleh $\left.\mu_{F\left(\varepsilon_{j}\right)}\left(u_{i}\right)\right)=\gamma_{F\left(\varepsilon_{j}\right)}\left(u_{i}\right)=0$.

$(\Longleftarrow)$ Misal $\mu_{F\left(\varepsilon_{j}\right)}\left(u_{i}\right)=\gamma_{F\left(\varepsilon_{j}\right)}\left(u_{i}\right)=0, \forall \varepsilon_{j} \in E, \forall u_{i} \in U$, maka akan dibuktikan $I(\omega)=0$.

Berdasarkan kondisi (b), diperoleh $\Phi\left(\mu_{F\left(\varepsilon_{j}\right)}\left(u_{i}\right), \gamma_{F\left(\varepsilon_{j}\right)}\left(u_{i}\right)\right)=0$.

Selanjutnya,

$$
\begin{aligned}
I(\omega) & =\sum_{j=1}^{n} \sum_{i=1}^{m}\left(1-\Phi\left(\mu_{F\left(\varepsilon_{j}\right)}\left(u_{i}\right), \gamma_{F\left(\varepsilon_{j}\right)}\left(u_{i}\right)\right)\right) \\
I(\omega) & =\sum_{j=1}^{n} \sum_{i=1}^{m}(1-0) \\
I(\omega) & =\sum_{j=1}^{n} \sum_{i=1}^{m}(1) \\
I(\omega) & =m n .
\end{aligned}
$$

(c) Akan dibuktikan $I(\omega)=I\left(\omega^{c}\right)$.

Ambil $\omega \in \mathcal{I F} \mathcal{S S}(\mathcal{U})$.

Misal $\left.\omega^{c}=\langle F, E\rangle^{c}=\left\langle F^{c},\right\rceil E\right\rangle$, dimana

$\left.F^{c}(\varepsilon)=\left\{\left(u_{i}, \gamma_{F\left(\varepsilon_{j}\right)}\left(u_{i}\right), \mu_{F\left(\neg \varepsilon_{j}\right)}\left(u_{i}\right)\right) \mid u_{i} \in U, \forall\left(\neg \varepsilon_{j}\right) \in\right\rceil E\right\}$.

Jika $I(\omega)=\sum_{j=1}^{n} \sum_{i=1}^{m}\left(1-\Phi\left(\mu_{F\left(\varepsilon_{j}\right)}\left(u_{i}\right), \gamma_{F\left(\varepsilon_{j}\right)}\left(u_{i}\right)\right)\right)$,

berdasarkan Definisi 2.5.1(c),

maka

$I\left(\omega^{c}\right)=\sum_{j=1}^{n} \sum_{i=1}^{m}\left(1-\Phi\left(\gamma_{F\left(\varepsilon_{j}\right)}\left(u_{i}\right), \mu_{F\left(\varepsilon_{j}\right)}\left(u_{i}\right)\right)\right)$.

Selanjutnya, berdasarkan kondisi (c), diperoleh

$\Phi\left(\mu_{F\left(\varepsilon_{j}\right)}\left(u_{i}\right), \gamma_{F\left(\varepsilon_{j}\right)}\left(u_{i}\right)\right)=\Phi\left(\gamma_{F\left(\varepsilon_{j}\right)}\left(u_{i}\right), \mu_{F\left(\varepsilon_{j}\right)}\left(u_{i}\right)\right)$, sehingga $I(\omega)=I\left(\omega^{c}\right)$.

(d) Akan dibuktikan $I(\omega) \geq I(\sigma)$.

Ambil $\sigma \in \mathcal{I F} \mathcal{S S}(\mathcal{U})$.

Misal $\sigma=\langle G, E\rangle=\left[b_{i j}\right]_{m \times n}$.

$\forall u_{i} \in U, \varepsilon_{j} \in E$, berdasarkan kondisi (d),

perhatikan bahwa:

$$
\begin{aligned}
& \Phi\left(\mu_{F\left(\varepsilon_{j}\right)}\left(u_{i}\right), \gamma_{F\left(\varepsilon_{j}\right)}\left(u_{i}\right)\right) \leq \Phi\left(\mu_{G\left(\varepsilon_{j}\right)}\left(u_{i}\right), \gamma_{G\left(\varepsilon_{j}\right)}\left(u_{i}\right)\right) \\
& 1-\Phi\left(\mu_{F\left(\varepsilon_{j}\right)}\left(u_{i}\right), \gamma_{F\left(\varepsilon_{j}\right)}\left(u_{i}\right)\right) \geq 1-\Phi\left(\mu_{G\left(\varepsilon_{j}\right)}\left(u_{i}\right), \gamma_{G\left(\varepsilon_{j}\right)}\right) \\
& \sum_{j=1}^{n} \sum_{i=1}^{m}\left(1-\Phi\left(\mu_{F\left(\varepsilon_{j}\right)}\right)\right)\left.\left.\left(u_{i}\right), \gamma_{F\left(\varepsilon_{j}\right)}\left(u_{i}\right)\right)\right) \\
& I(\omega) \geq \sum_{j=1}^{n} \sum_{i=1}^{m}\left(1-\Phi\left(\mu_{G\left(\varepsilon_{j}\right)}\left(u_{i}\right), \gamma_{G\left(\varepsilon_{j}\right)}\left(u_{i}\right)\right)\right) \\
& I(\sigma)
\end{aligned}
$$

Karena $\Phi$ memenuhi kondisi (a),(b),(c), dan (d), maka I merupakan sebuah entropi. 
24 Raisatul Mardhiyah, dkk

\section{Kesimpulan}

Pada jurnal ini telah dijelaskan hubungan antara himpunan lembut kabur intuisionistik dan himpunan lembut kabur merupakan suatu fungsi dan memenuhi sifat-sifat terkaitnya. Disamping itu, telah dibahas juga suatu sifat dan contoh entropi pada himpunan lembut kabur intuisionistik.

\section{Ucapan Terima kasih}

Penulis mengucapkan terima kasih kepada bapak Dr. Admi Nazra, ibuk Nova Noliza Bakae, M.Si, dan bapak Prof. Dr. I Made Arnawa selaku dosen penguji tugas akhir yang telah memberikan masukkan dan saran sehingga makalah ini dapat diselesaikan dengan baik.

\section{Daftar Pustaka}

[1] Atanassov,K.T. 1986. Intuitionistic Fuzzy Sets. Fuzzy Sets and Systems. 20: $87-96$.

[2] Atanassov,K.T. 1988. Two Operators on Intuisionistic Fuzzy Sets. Comptes Rendus de l'Acadkmi Buloare des Sciences. 41(5): 35-38.

[3] Atanassov,K.T., Gargov.G 1989. Interval Valued Intuitionistic Fuzzy Sets Fuzzy Sets and Systems. 31: 343-349.

[4] Burillo,P., Bustince,H. 1994. Entropy on Intuitionistic Fuzzy Sets and on Interval Valued Fuzzy Sets. Fuzzy Sets and Systems. 78: 305-316.

[5] Burillo,P., Bustince,H. 1996. Construction Theorems for Intuitionistic Fuzzy Sets. Fuzzy sets and Systems. 84: 271-281.

[6] Cagman,N., Enginoglu,S. 2010. Soft Matrix Theory and its Decison Making. Computers and Mathematics with Application. 59(10): 3308-3314.

[7] Jiang, Y. et.al. 2013. Entropy on Intuitionistic Fuzzy Soft Sets and on IntervalValued Fuzzy Soft Sets. Information Sciences. 240: 95-114.

[8] Maji,P.K., Biswas,R., Roy,A.R. 2001. Fuzzy Soft Sets. J. Fuzzy Math. 9(3): 589-602.

[9] Maji,P.K., Biswas,R., Roy,A.R. 2009. More on Intuitionistic Fuzzy Soft Sets. Comput. Math. Appl. 45: 555-562.

[10] Molodtsov,D.A. 1999. Soft Set Theory First-Result. Comput. Math. Appl. 37: 19-31.

[11] Zadeh,L.A. 1965. Fuzzy sets. inf and control. 8: 338-353. 